\title{
Drawing the line between a normal and mildly abnormal nuclear cardiology scan
}

\author{
Todd D. Miller, MD, MASNC ${ }^{\mathrm{a}}$ \\ a Mayo Clinic, Rochester, MN
}

Received Dec 2, 2020; accepted Dec 2, 2020

doi: $10.1007 /$ s12350-020-02477-7

\section{See related article, pp. 826-835}

Telephone conversation between nuclear cardiologist and general internist at the end of a busy work day:

Nuclear cardiologist: Hi Mary. This is Mike. Mrs. Smith's nuclear images look pretty good.

Internist: Oh great! She will be reassured to hear that her images are normal.

Nuclear cardiologist: Well, they aren't quite normal.

Internist: Oh. So what do you see?

Nuclear cardiologist: There is a small mild apical perfusion defect that appears slightly reversible. These findings are more evident in the apical short-axis and horizontal long-axis views than in the vertical long-axis views.

Internist: So what do you think this defect represents?

Nuclear cardiologist: Not sure. It might represent a small area of ischemia, but it could also simply be artifact from shifting breast shadow.

Internist: So what should I tell Mrs. Smith?

This clinical scenario highlights one of the common challenges encountered in nuclear cardiology. The accuracy of myocardial perfusion imaging (MPI) is limited due to imaging artifact, which usually causes mild abnormalities. Additionally, significant obstructive coronary artery disease (CAD) can also result in mild perfusion abnormalities. The differentiation between a

\footnotetext{
Reprint requests: Todd D. Miller, MD, MASNC, Mayo Clinic, 200

First Street, SW, Rochester, MN 55905; miller.todd@mayo.edu J Nucl Cardiol 2022;29:836-9.

$1071-3581 / \$ 34.00$

Copyright (c) 2021 American Society of Nuclear Cardiology.
}

normal and a mildly abnormal study remains a challenging problem.

The standard for interpreting and reporting MPI applies a 17-segment model of the left ventricle with perfusion graded in each segment using a 5-point scale. ${ }^{1}$ The summation of the perfusion grades on the stress images is the summed stress score (SSS) and on the rest images the summed rest score (SRS). SSS represents the extent and severity of combined ischemia and infarction, and SRS represents the extent and severity of infarction. The difference between SSS and SRS is the summed difference score (SDS), a measure of the extent and severity of ischemia. ${ }^{1}$ The Cedars-Sinai laboratory introduced and popularized this summed scoring scheme. $^{2,3}$ Realizing that imaging artifact accounts for many mildly abnormal scans, they required in their initial studies that an abnormal stress image had to have two or more segments with perfusion scores $\geq 2$ (at least moderately reduced isotope uptake). Thus, a normal SSS was not limited to only SSS $=0$ but to a score range of 0-3. An ischemic defect was defined as moderately reduced uptake on the stress images (grading $\geq 2$ ) with a rest score $\leq 1$, or a stress defect with absent uptake (grading 4) with a rest score of 2. Thus, an ischemic image had $\mathrm{SDS} \geq 2$. Subsequent prognostic studies from the Cedars-Sinai laboratory demonstrated that patients with normal or mildly normal MPI (SSS 0-3) constituted a low-risk group, with annual risk of cardiac death or non-fatal myocardial infarction (MI) $<1 \%$. $^{4,5}$ These findings were extensively validated by many other nuclear cardiology laboratories with similar results. ${ }^{6}$

The concept of a low-risk scan is well-established, and there has been little investigation of prognosis with normal versus mildly abnormal MPI. In this issue of JNC, Kassab et al. ${ }^{7}$ examine the prognostic significance of completely normal MPI compared to mildly abnormal images or borderline myocardial ischemia. The study population consisted of 6802 patients who underwent regadenoson SPECT MPI over a 6-year period. Normal 
scans were present in 4398 patients and near-normal scans in 2404 patients. All patients had normal left ventricular ejection fraction $(\mathrm{LVEF}) \geq 50 \%$. Normal MPI was defined as SSS $=0$ and near-normal MPI as SSS $=1-3$. Among the 2404 patients with near-normal MPI, 972 also had borderline ischemia, defined as $\mathrm{SDS}=1$. During mean follow-up of $2.5 \pm 2.1$ years, there were 158 hard cardiac events (combined cardiac deaths and non-fatal MIs). Coronary revascularizations were performed in 246 patients, which included 96 early (within 90 days of MPI) and 150 late (beyond 90 days) procedures. The major finding is that neither near-normal MPI nor borderline ischemia identified patients at higher risk for the endpoint of cardiac death/non-fatal MI: near-normal MPI versus normal MPI (HR 1.21; 95\% CI 0.88-1.66; $P=0.24)$; borderline ischemia versus no ischemia (HR 1.09; 95\% CI 0.70-1.69; $P=0.69$ ). There were significantly more coronary revascularizations performed in patients with near-normal MPI (HR $1.91 ; 95 \%$ CI 1.49-2.46; $P<0.001)$ and borderline ischemia (HR 5.62; 95\% CI 3.08-10.25; $P<0.001$ ). Late coronary revascularizations were also higher in patients with borderline ischemia (HR 2.98; 95\% CI 1.36-6.53; = 0.006).

How should these results be interpreted? The most important observation is the absence of an association between mildly abnormal MPI and the composite endpoint of cardiac death/MI, which the authors selected as their primary endpoint. The premise for performing this study was that mildly abnormal MPI might identify a significant number of patients with prognostically important CAD and not just imaging artifact. If correct, this subset of patients would be expected to have a higher event rate than the subset with completely normal MPI. This type of result could lead to a recommendation to separate patients with normal and patients with mildly abnormal MPI into different risk categories, for both clinical practice and research purposes. However, the negative results by Kassab et al suggest that there is no reason to change the thresholds of SSS $\leq 3$ and SDS $\leq 1$ for identification of low-risk patients. Further subcategorization does not appear to be helpful for prognostic purposes.

The other major result from this study is the positive association between both near-normal MPI and borderline ischemia with coronary revascularization. The authors examined all revascularization procedures and separately the category late revascularization procedures. The authors selected revascularization to represent CAD burden, although no data were presented to support this assumption. It should be appreciated that revascularization procedures reflect clinical decisionmaking and differ substantially from spontaneous coronary events of death and $\mathrm{MI}$, which represent the natural history of CAD. The traditional paradigm for evaluating and managing chronic CAD includes the identification of significant ischemia, followed by coronary angiography and revascularization, based upon the presumption that this approach would lower the risk of cardiac death/MI. Adhering to this paradigm should result in a significant association between clearly abnormal MPI and early revascularization. As such it might be anticipated that even mildly abnormal MPI might be associated with early revascularization (not analyzed separately by Kassab et al, but incorporated into their total revascularization analysis). Prior studies have demonstrated that nuclear imaging variables, especially ischemia, are very strong predictors of referral to early coronary angiography. ${ }^{8,9}$ In this study even borderline ischemia (SDS =1) was associated with a more than five-fold increase in revascularization. Mildly abnormal findings on MPI are commonly accompanied in nuclear cardiology reports by subjective statements which include terms such as "equivocal", "borderline', and " probably". Although such terms are applied by the nuclear cardiologist to reflect some uncertainty with image interpretation, these terms may create in the mind of the ordering clinician and/or patient a "hedged" result that requires coronary angiography for definitive resolution. The net result is that some patients with mildly abnormal MPI will be identified as having obstructive CAD and undergo revascularization.

The authors analyzed late coronary revascularization separately, under the assumption that it represents CAD progression or severe CAD burden that failed conservative management. The concept of late revascularization was introduced as an endpoint in studies performed more than 30 years ago that were relatively small by today's standards. ${ }^{10,11}$ Late revascularization was created to increase the number of "events" and enhance the statistical power of a study. The reason for selecting a threshold of 60 or 90 days to separate early from late revascularization was that revascularization procedures being performed in response to the nuclear test results could generally be scheduled within this timeframe. If revascularization could not be performed within this window, it likely was being done for subsequent progression of CAD. Another assumption was that revascularization could alter the natural history of CAD and avert an MI or cardiac death that would have otherwise occurred. Unfortunately, equating late revascularization to a surrogate for a hard cardiac event is based upon these assumptions, which have never been proven true. Some late revascularizations are performed in stable patients, in whom both the patient and clinician are aware of and influenced by the results of the nuclear stress test, even if performed more than 60 or 90 days ago. Additionally, revascularization in the setting of 
chronic CAD has not been shown to decrease cardiac events. $^{12,13}$ In studies that have adequate statistical power (158 hard events in the current study), revascularization procedures should be reported for descriptive purposes but should not be included in the formal statistical analyses examining the association between test results with clinical outcome.

Important methodological limitations of this study should be noted. The stress modality in all patients was regadenoson. The rationale for excluding patients who underwent exercise stress is not provided. Patients with LVEF $<50 \%$ were excluded. The results of this study may not apply to patients who undergo exercise stress or who have left ventricular systolic dysfunction. The images were interpreted solely by a computer software program (INVIA Corridor 4DM-Ann Arbor, MI). Most nuclear cardiology laboratories use an integrative approach, combining subjective reading of the images with the results generated by a computer software program, often followed by review of the clinical record, to issue a final report. This approach can generate a report that is substantially different from the results produced by the computer software program alone. The study population was obese (mean BMI 32), and attenuation correction was not applied. This issue is particularly relevant to a study evaluating the significance of mild MPI abnormalities. The completeness of collection of outcome data is not provided. The determination of death should be accurate, as the SSDI was applied. The cause of death could be determined in all but 9 patients. However, non-fatal MI was only determined in patients who were subsequently hospitalized at Rush University. In a city the size of Chicago, it is likely that some patients had an MI and were hospitalized elsewhere. A uniform definition of MI was not applied. The authors do not identify the individual(s) who made this determination, nor do they address the issue of blinding of this individual(s) to the results of MPI.

As noted by Kassab et al, there has been little prior investigation of this issue. Abidov et $\mathrm{al}^{14}$ investigated the prognostic value of MPI interpretation using 5 categories versus a simple dichotomous normal/abnormal classification using a 20-segment model. For the 3 lowest risk categories coded "normal" (all segments coded 0 , or $\leq 2$ segments coded 1-1), "probably normal" ( $\geq 3$ segments coded $1-1$, or 1 segment coded 1 0 ), or "equivocal" ( 2 or 3 segments coded $1-0$, or 1 segment coded 2-1 or 2-0), annual cardiac death rates were significantly higher $(P<0.001)$ in the equivocal category. However, event rates were low for all 3 groups at $0.4 \%, 0.5 \%$, and $1.0 \%$, respectively. In the REFINE SPECT registry, ${ }^{15}$ the investigators applied both subjective and quantitative assessment of MPI to evaluate the prognostic value of newer high-efficiency SPECT scanners. The subjective analysis was based upon 4 categories of SSS scores: normal $(\mathrm{SSS}=0)$, probably normal ( $\mathrm{SSS}=1$ ), equivocal ( $\mathrm{SSS}=2-3$ ), abnormal $(\mathrm{SSS} \geq 4)$. Quantitative analysis applied Quantitative Perfusion SPECT software (Cedars Sinai Medical Center, Los Angeles, CA) to generate total perfusion deficit (TPD) as a continuous variable, with subsequent creation of 4 categories to approximate the 4 subjective categories: normal (TPD $0 \%-<1 \%$ ), probably normal (TPD $\geq 1 \%-<3 \%)$, equivocal $\quad(\mathrm{TPD} \geq 3 \%-<5 \%)$, and abnormal (TPD $\geq 5 \%$ ). There was a statistically significant worsening prognosis across increasingly more severe stress MPI categories, including differences between the 3 lowest risk categories (normal, probably normal, equivocal) for the authors' selected primary endpoint of MACE (all-cause mortality, non-fatal MI, unstable angina, or late coronary revascularization). Among the 2760 MACE, 1025 were late revascularizations. In another analysis limited to using the endpoint of overall mortality/non-fatal MI and patients categorized by both subjective analysis and TPD, there were few statistical differences between categories for the 3 lowest risk groups. The annual hard event rate for patients in the reference group (both stress TPD $<1$ and SSS $=0$ ) was $1.1 \%$. For patients with TPD categories $(\geq 1$ but $<3$ ) and $(\geq 3$ but $<5$ ), event rates were nearly as low, between $1.3 \%-1.8 \%$, for patients with subjective $\mathrm{SSS}=0$ or $\mathrm{SSS}=1$. The event rate only became meaningfully higher at $2.4 \%-2.5 \%$ for patients in these TPD categories who also had subjective SSS $=2-3$.

In conclusion, the results from the limited literature on this topic do not provide a compelling reason to subdivide the low-risk SSS 0-3 category into sub-categories. There is little difference in outcome for patients with a normal scan versus a mildly abnormal scan when the outcome endpoint being analyzed is hard cardiac events. Perhaps not surprising is that mildly abnormal scans are associated with higher rates of both early and late revascularization, likely reflecting clinicians' discomfort with equivocal or borderline results and a lower threshold to proceed with coronary angiography. The clinical importance of this observation is questionable, given that recent studies have failed to demonstrate a reduction in hard cardiac events with revascularization in patients with chronic CAD. ${ }^{12,13}$ Where to set the threshold to separate a normal from a mildly abnormal study represents the classic trade-off between test sensitivity and specificity. Kassab et al. conclude that subtle perfusion abnormalities should not be ignored. Although their advice is prudent, the nuclear cardiologist should also not mislead the clinician into thinking that all mild abnormalities are clinically important. Such a strategy would lead to a modest increase in test sensitivity but at the expense of a substantial reduction in test specificity. 
Any future study that addresses this issue should focus on two goals: (1) demonstrating a statistically significant, clinically meaningful difference in hard cardiac events for mildly abnormal versus normal images; and (2) performing a careful cost analysis of downstream resource use that results from labeling mild or borderline MPI findings as definitely abnormal. Identifying more patients with CAD who could potentially benefit from intensified preventive strategies is a laudable goal. However, generating more patients for referral to coronary angiography who do not have obstructive CAD or only have a borderline significant coronary stenosis potentially undermines the referring doctor's confidence in MPI, exposes the patient to a small but finite risk by proceeding with coronary angiography, and wastes health care dollars if clinical outcome is not improved. All of these issues need to be carefully evaluated before the threshold identifying an abnormal from a normal MPI scan is shifted.

\section{Disclosure}

The author has indicated that he has no financial conflict of interest.

\section{References}

1. Tilkemeier PL, Bourque J, Doukky R, Sanghani R, Weinberg RL. ASNC imaging guidelines for nuclear cardiology procedures. J Nucl Cardiol. 2017;24:2064-128.

2. Berman DS, Kiat H, Van Train K, Garcia E, Friedman J, Maddahi J. Technetium $99 \mathrm{~m}$ sestamibi in the assessment of chronic coronary artery disease. Semin Nucl Med. 1991;21:190-212.

3. Berman DS, Kiat H, Friedman JD, Wang FP, van Train K, Matzer L, et al. Separate acquisition rest thallium-201/stress technetium$99 \mathrm{~m}$ sestamibi dual-isotope myocardial perfusion single-photon emission computed tomography: a clinical validation study. J Am Coll Cardiol. 1993;22:1455-64.

4. Berman DS, Hachamovitch R, Kiat H, Cohen I, Cabico JA, Wang $\mathrm{FP}$, et al. Incremental value of prognostic testing in patients with known or suspected ischemic heart disease: a basis for optimal utilization of exercise technetium- $99 \mathrm{~m}$ sestamibi myocardial perfusion single-photon emission computed tomography [published erratum appears in J Am Coll Cardiol 1996 Mar 1;27(3):756]. J Am Coll Cardiol. 1995;26:639-47.
5. Hachamovitch R, Berman DS, Kiat H, Cohen I, Cabico JA, Friedman $\mathrm{J}$, et al. Exercise myocardial perfusion SPECT in patients without known coronary artery disease: Incremental prognostic value and use in risk stratification. Circulation. 1996;93:905-14.

6. Shaw LJ, Iskandrian AE. Prognostic value of gated myocardial perfusion SPECT. J Nucl Cardiol. 2004;11:171-85.

7. Kassab K, Iskander F, Iskander M, Khan R, Doukky R. Prognostic value of near normal perfusion or borderline ischemia on stress myocardial perfusion imaging. J Nucl Cardiol. 2020. https://doi. org/10.1007/s12350-020-02375-y.

8. Miller TD, Roger VL, Hodge DO, Hopfenspirger MR, Bailey KR, Gibbons RJ. Gender differences and temporal trends in clinical characteristics, stress test results and use of invasive procedures in patients undergoing evaluation for coronary artery disease. J Am Coll Cardiol. 2001;38:690-7.

9. Hachamovitch R, Hayes SW, Friedman JD, Cohen I, Kang X, Germano $\mathrm{G}$, et al. Is there a referral bias against catheterization of patients with reduced left ventricular ejection fraction?: Influence of ejection fraction and inducible ischemia on post-single-photon emission computed tomographymanagement of patients without a history of coronary artery disease. J Am Coll Cardiol. 2003;42:1286-94.

10. Gill JB, Ruddy TD, Newell JB, Finkelstein DM, Strauss HW, Boucher CA. Prognostic importance of thallium uptake by the lungs during exercise in coronary artery disease. $\mathrm{N}$ Engl $\mathrm{J}$ Med. 1987;317:1485-9.

11. Kaul S, Lilly DR, Gascho JA, Watson DD, Gibson RS, Oliner CA, et al. Prognostic utility of the exercise thallium-201 test in ambulatory patients with chest pain: comparison with cardiac catheterization. Circulation. 1988;77:745-58.

12. Stergiopoulos K, Boden WE, Hartigan P, Möbius-Winkler S, Hambrecht R, Hueb W, et al. Percutaneous coronary intervention outcomes in patients with stable obstructive coronary artery disease and myocardial ischemia: a collaborative meta-analysis of contemporary randomized clinical trials. JAMA Intern Med. 2014;174:232-40.

13. Maron DJ, Hochman JS, Reynolds HR, Bangalore S, O'Brien SM, Boden WE, et al. Initial invasive or conservative strategy for stable coronary disease. N Engl J Med. 2020;382:1395-407.

14. Abidov A, Hachamovitch R, Hayes SW, Friedman JD, Cohen I, Kang $\mathrm{X}$, et al. Are shades of gray prognostically useful in reporting myocardial perfusion single-photon emission computed tomography? Circ Cardiovasc Imaging. 2009;2:290-8.

15. Otaki Y, Betancur J, Sharir T, Hu L-H, Gransar H, Liang J, et al. 5 -year prognostic value of quantitative vs visual MPI in subtle perfusion defects. Results from REFINE SPECT. J Am Coll Cardiol. 2020;13:774-85.

Publisher's Note Springer Nature remains neutral with regard to jurisdictional claims in published maps and institutional affiliations. 